\title{
Coelacanths from the Middle Triassic Luoping Biota, Yunnan, South China, with the earliest evidence of ovoviviparity
}

Wen Wen, Qi-Yue Zhang, Shi-Xue Hu, Michael J. Benton, Chang-Yong Zhou, Xie Tao, Jin-Yuan Huang, and Zhong-Qiang Chen

Acta Palaeontologica Polonica 58 (1), 2013: 175-193 doi: http://dx.doi.org/10.4202/app.2011.0066

The fossil record of coelacanths is patchy, with very few taxa known from the Triassic of Asia. We report here two new genera and species of coelacanths from the Luoping Biota, a recently found site of exceptional fossil preservation from Yunnan, South China. The first new taxon, Luopingcoelacanthus eurylacrimalis, is based on four specimens, which together show most aspects of the anatomy. One specimen shows two small coelacanths inside the ventral portion of the abdominal cavity, and these are interpreted as intrauterine embryos, close to birth size, based on comparisons with previously reported embryos of the fossil coelacanths Rhabdoderma and Undina, and the extant genus Latimeria. Our new find extends the evidence for ovoviviparity in coelacanths back from the Late Jurassic to the Middle Triassic. The second new taxon, Yunnancoelacanthus acrotuberculatus, is based on one specimen, and differs from Luopingcoelacanthus in the dentary, lachrymojugal, number of rays of the first dorsal fin, and especially in the ornament on dermal bones and scales. Acladistic analysis shows that the new taxa are closest relatives to the derived clade Latimerioidei. The relatively high diversity of coelacanths in the Early Triassic, and adaptations of living Latimeria to low-oxygen conditions, suggests that the group may have included 'disaster taxa' that benefited from anoxic and dysoxic ocean conditions in the aftermath of the end-Permian mass extinction.

Key words: Actinistia, Coelacanthoidei, Latimerioidei, ovoviviparity, Luoping, Anisian, Triassic, South China.

Wen Wen [wenwen2020240@163.com], Qi-Yue Zhang [yxzqy@sina.com],

Shi-Xue Hu [hushixue@hotmail.com], Chang-Yong Zhou [zhcy79@163.com], Xie

Tao [xt1982cd@163.com], Jin-Yuan Huang [huangjinyuancug@gmail.com

], Chengdu Institute of Geology and Mineral Resources, Chengdu 610081,

Chengdu University of Technology, Chengdu 610059, China; Michael J.

Benton [mike.benton@bristol.ac.uk], School of Earth Sciences,

University of Bristol, Bristol BS8 1RJ, UK; Zhong-Qiang Chen [zhong.qiang.chen@uwa.edu.au] State

Key Laboratory of Biogeology and Environmental Geology, China University of Geosciences (Wuhan), 
Wuhan 430074, China.

This is an open-access article distributed under the terms of the Creative Commons Attribution License (for details please see creativecommons.org), which permits unrestricted use, distribution, and reproduction in any medium, provided the original author and source are credited.

For Full text $(2,553.0 \mathrm{kB})$ 